\title{
Intensity, Phase and Polarization of Vector Bessel Vortex Beam Through Multilayered Isotropic Media
}

\author{
HAIYING LI, ${ }^{1, *}$ FARIDEh Honary, ${ }^{2}$ JiAJIE WANG, ${ }^{1}$ JIAWEI LIU, ${ }^{1}$ ZhenSEN Wu, ${ }^{1,{ }^{*}}$ LU \\ BAI, ${ }^{1}$
}

${ }^{1}$ School of Physics and Optoelectronic Engineering, Xidian University, Xi'an, 710071, China

${ }^{2}$ Department of Physics, Lancaster University, Lancaster, LA1 4YB, UK

*Corresponding author: lihy@xidian.edu.cn,wuzhs@mail.xidian.edu.cn

Received XX Month XXXX; revised XX Month, XXXX; accepted XX Month XXXX; posted XX Month XXXX (Doc. ID XXXXX); published XX Month XXXX

This paper investigates the characteristics of reflected and transmitted fields of a vector Bessel vortex beam through multilayered isotropic media on the basis of the vector angular spectrum expansion, and presents the effects of media on intensity, phase and polarization. The method is verified by studying the reflection and transmission on a single interface at the vertical incidence. For both paraxial and non-paraxial incident beam cases, numerical simulations of the field components and the time-averaged Poynting vector power density of the reflected and the transmitted beams for the three-layered media are presented and discussed in detail. It is shown that as the incident angle increases, the magnitude distribution of the reflected beams illustrates significant distortions and no longer represents similar patterns to that of incident beam, whereas the magnitude distribution of the transmitted beams can maintain similar profiles to the incident beam, apart from the notable distortion of the central ring. For the same incident angle, the effects of media on the magnitude distribution for the nonparaxial case are more evident than those for the paraxial case. The results of phase distribution and polarization of the reflected and transmitted fields show that as the incident angle increases, the distortion of the phase distribution and polarization for the reflected fields are more significant, and the topological charge cannot preserve. (c) 2015 Optical Society of America

OCIS codes: (140.3295) Laser beam characterization; (080.2710) Inhomogeneous optical media; (070.2465) Finite analogs of Fourier transforms. http://dx.doi.org/10.1364/AO.99.099999

\section{INTRODUCTION}

Bessel beams were firstly put forward as non-diffracting, exact nonsingular solutions of the scalar wave equation in 1987 [1], whose field amplitudes can be described by Bessel function of the first kind. Although the ideal Bessel beams with infinite transverse extent and energy cannot be produced experimentally, the quasi-Bessel beams with finite size approximation can be generated by use of holographic elements [2], an axicon [3, 4, 5], a Spatial Light Modulator (SLM) [6], a conical mirror [7] or a Digital Micro Mirror device (DMD) [8]. Because of their special properties, such as non-diffraction [1], selfreconstruction [9] and superluminality [10], Bessel beams have prospective applications in the fields of optical manipulation [11], the design of optics devices, imaging [12] and communication [13]. Most noteworthy, investigation results show that beams with orbital angular momentum (OAM), which are also called vortex beams, have great potential in improving the communication efficiency [14-16], hence the high-order Bessel beams [17-19], which belong to a class of OAM beams, are worthy of more attention.
Understanding the effects of various media on Bessel beam propagation is an important premise to study the potential applications of Bessel beams. Description of vector Bessel beams has been provided by use of Hertz vector potentials and angular spectrum expansion [20-25]. The interactions of other beams (including Gaussian beam, Laguerre-Gaussian beam) with media [26-33] have been investigated, especially the reflection and transmission on a dielectric interface, or a slab structure through angular spectrum expansion [26-30], two-dimensional Finite-Difference Time-Domain (FDTD) approach [31], and Vector Cylindrical Wave Functions (VCWFs) expansion of beams $[32,33]$ for the past two decades. For the Bessel beam, Aiello studied the Goos-Hänchen (GH) and the Imbert-Fedorov (IF) shifts with vector angular spectrum expansion [34]. Mugnai analyzed the transmission through a slab in both scalar and vectorial approximations [35], for the case of total reflection [36], as well as for the case of reflection from the conducting and absorbing media [37, 38]. Novitsky studied the intensity transformation of a Bessel beam at total internal reflection for a dielectric interface [39], as well as the change of the size of vector Bessel beam rings under reflection [40].

As far as the multilayered or inhomogeneous media, which are usually found in optical film technique, optical devices, and optical 
communication fields, are concerned, although the reflection and transmission of a plane wave in multilayered or inhomogeneous media can be analyzed by the well-known Snell's law and Fresnel formulas, for the propagation of beams with a finite width, the problem becomes complex, for example, Snell's law and Fresnel formulas cannot be used directly, and some special properties are exhibited in the reflected and transmitted fields, including the nonspecular phenomena [41], the lateral displacements [42], and GH shifts [43]. With regard to the propagation of Bessel beams in multilayered or inhomogeneous media, Manela identified the intensity and phase properties in twodimensional periodic systems [45]. Novitsky investigated the reflection and transmission spectra by the matrix approach and mainly discussed the dependence of reflectivity on the normalized frequency and the normalized radius in bi-anisotropic media [46]. With FDTD and angular spectrum method, Elmaklizi studied the propagation of Bessel beam in scattering media [47], while Zheng also did this work using the angular spectrum method combined with slice-by-slice propagation model [48]. Although some research on the propagation of Bessel beams in inhomogeneous media is available in the literature, the reflected and transmitted field characteristics of Bessel vortex beams from multilayered isotopic media by vector angular spectrum expansion method haven't been reported systematically, which is the purpose of this work.

The structure of the paper is as follows. The physical model, the description and the numerical simulations of the incident high-order Bessel vortex beam are presented in Section2.A and Section2.B, respectively. The reflected and transmitted electric fields are expressed by the vector angular spectrum in Section 3, as well as the polarization is also described in this section. The validity of the method proposed in the paper, the magnitude distribution of the field components, the time-averaged Poynting vector power density, the phases and the polarization for both reflected and transmitted beams are discussed in Section4. Finally, Section 5 draws the main conclusions of our work.

\section{THEORETICAL BACKGROUNDS}

\section{A. Physical model}

An incident vector Bessel vortex beam is considered on the interface of air and N-layers isotropic media, whose physical model is shown in Fig.1. The $x-y$ plane of the global coordinate $\operatorname{system}(x, y, z)$ is situated on the first interface $(z=0)$, and the principal incident plane is the $x-z$ plane. The local coordinate systems $\left(x_{\beta}, y_{\beta}, z_{\beta}\right)$ $\beta=i, r$, or $t$ are attached to the incident, the reflected and the transmitted beams, respectively, where the positive directions of the $z_{\beta}$ axes are parallel to the central propagation directions of them. The Cartesian unit vectors in the global coordinate system can be written as $\hat{x}=\hat{x}_{i} \cos \theta+\hat{z}_{i} \sin \theta, \hat{y}=\hat{y}_{i}$ and $\hat{z}=-\hat{x}_{i} \sin \theta+\hat{z}_{i} \cos \theta$, respectively. The angle between $z_{i}$ and the $z$ axes is defined as the incident angle, which is denoted as $\theta$. Similarly, the angles $\theta_{r}$ and $\theta_{t}$ represent the reflection and the transmission angles, respectively.

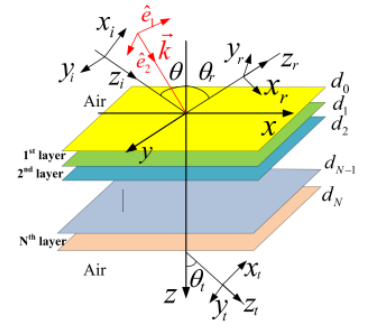

Fig.1. Schema of vector Bessel vortex beam incident on N-layers media.
In Fig.1, the coordinate values of the interfaces in the global coordinate system are $z=d_{0}, d_{1}, \ldots, d_{N-1}, d_{N}$. The refractive index and the wave number in the $j$ th region are $n_{j}$ and $k_{j}$, respectively. The magnetic susceptibility of each region is assumed to be unity. $\vec{k}$ with $|\vec{k}|=k_{0}$ denotes the wave vector of the incident Bessel beam and can be expressed as

$$
\vec{k}=k_{i x} \hat{x}_{i}+k_{i y} \hat{y}_{i}+k_{i z} \hat{z}_{i}=k_{x} \hat{x}+k_{y} \hat{y}+k_{z} \hat{z},
$$

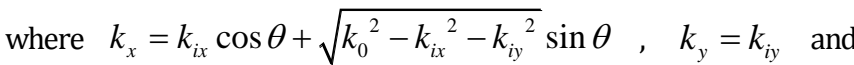

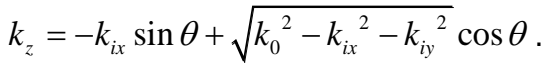

\section{B. Description of the incident Bessel Vortex beams}

The electric field of a scalar Bessel vortex beam is:

$$
E_{i}\left(r_{i}, \varphi_{i}, z_{i}\right)=E_{0} J_{n}\left(k_{i t 0} r_{i}\right) e^{i n \varphi_{i}} e^{i k_{i z} z_{i}},
$$

where $r_{i}=\sqrt{x_{i}^{2}+y_{i}^{2}}, \varphi_{i}=\arctan \left(y_{i} / x_{i}\right), k_{i t 0}=k_{0} \sin \vartheta_{0}$, $k_{i z}=k_{0} \cos \vartheta_{0}$ are the transverse and the longitudinal wave numbers, respectively. $\vartheta_{0}\left(0 \leq \vartheta_{0} \leq \pi / 2\right)$ is the half-cone angle, and $n>0$ is the topological charge. The beam is paraxial, when the inequality $\sin \vartheta_{0}<<1$ is met; for the non-paraxial beam this condition is not fulfilled. The time dependence factor of $\exp (-i \omega t)$ is omitted.

When the Bessel beam is incident on the interface with the angle $\theta$, with the help of angular spectrum of plane wave, the electric and the magnetic fields of the vector Bessel vortex beam in the local coordinate system can be written as:

$$
\begin{aligned}
\vec{E}_{i}\left(x_{i}, y_{i}, z_{i}\right)= & \frac{1}{2 \pi} \sum_{\lambda=1}^{2} \int \hat{e}_{\lambda}\left(\vec{k}_{i t}\right) \tilde{A}_{\lambda}\left(k_{i x}, k_{i y}\right) \\
& \exp \left(i\left(k_{i x} x_{i}+k_{i y} y_{i}+k_{i z} z_{i}\right)\right) d k_{i x} d k_{i y}, \\
\vec{B}_{i}\left(x_{i}, y_{i}, z_{i}\right)= & \frac{1}{2 \pi} \frac{\vec{k}}{\omega} \times \sum_{\lambda=1}^{2} \int \hat{e}_{\lambda}\left(\vec{k}_{i t}\right) \tilde{A}_{\lambda}\left(k_{i x}, k_{i y}\right) \\
& \exp \left(i\left(k_{i x} x_{i}+k_{i y} y_{i}+k_{i z} z_{i}\right)\right) d k_{i x} d k_{i y},
\end{aligned}
$$

where $\tilde{A}_{\lambda}\left(k_{i x}, k_{i y}\right)=\hat{e}_{\lambda}\left(\vec{k}_{i t}\right) \cdot \hat{f} \cdot \tilde{A}\left(k_{i x}, k_{i y}\right), k_{i t}=\left(k_{i x}{ }^{2}+k_{i y}{ }^{2}\right)^{1 / 2}$, $\tilde{A}\left(k_{i x}, k_{i y}\right)=E_{0} \delta\left(k_{i t}-k_{i t 0}\right) e^{i n \phi_{i}} / i^{n} k_{i t 0} \quad, \quad \vec{k}_{i t} \cdot \vec{r}_{i}=k_{i x} x_{i}+k_{i y} y_{i} ，$ $\left\{\hat{e}_{\lambda}\left(\vec{k}_{i t}\right)\right\}_{\lambda=1,2}$ are the polarization unit vectors of the angular spectrum [26], and the complex-valued unit vector $\hat{f}=f_{p} \hat{x}_{i}+f_{s} \hat{y}_{i}$ denotes the polarization of the incident beam, with $|\hat{f}|=1$. Then, the angular spectrum of vector vortex Bessel beam is $\bar{A}\left(k_{i x}, k_{i y}\right)=\sum_{\lambda=1}^{2} \hat{e}_{\lambda}\left(\vec{k}_{i t}\right) \tilde{A}_{\lambda}\left(k_{i x}, k_{i y}\right)$.

According to the definition of polarization unit basis vectors in Fourier space, $\left\{\hat{e}_{\lambda}\left(\vec{k}_{i t}\right)\right\}_{\lambda=1,2}$ are given in Appendix.

Considering the features of Bessel vortex beam, it is more convenient to convert Eq.(3) into spherical coordinate system $\left(k_{0}, \vartheta, \phi_{i}\right)$, that is

$$
\begin{aligned}
\vec{E}_{i}\left(\vec{r}_{i}\right)= & \frac{1}{2 \pi} \sum_{\lambda=1}^{2} \int \hat{e}_{\lambda} \tilde{A}_{\lambda}\left(\sin \vartheta \cos \phi_{i}, \sin \vartheta \sin \phi_{i}\right) . \\
& e^{i k_{0} r_{i} \sin \vartheta \cos \left(\phi_{i}-\varphi\right)} e^{i k_{0} \cos \vartheta z_{i}} \sin \vartheta \cos \vartheta d \vartheta d \phi_{i} .
\end{aligned}
$$


For the purpose of demonstration, by solving the integral expression in Eq. (5) with numerical method, the magnitude distributions of field components for both paraxial and non-paraxial Bessel beams are displayed in Figs.2, 3. The polarization unit vector $\hat{f}=\hat{x}_{i}$ and the beam order $n=2$ are considered. The half-cone angle for the paraxial Bessel beam is $10^{\circ}$, while for the non-paraxial case is $30^{\circ}$.
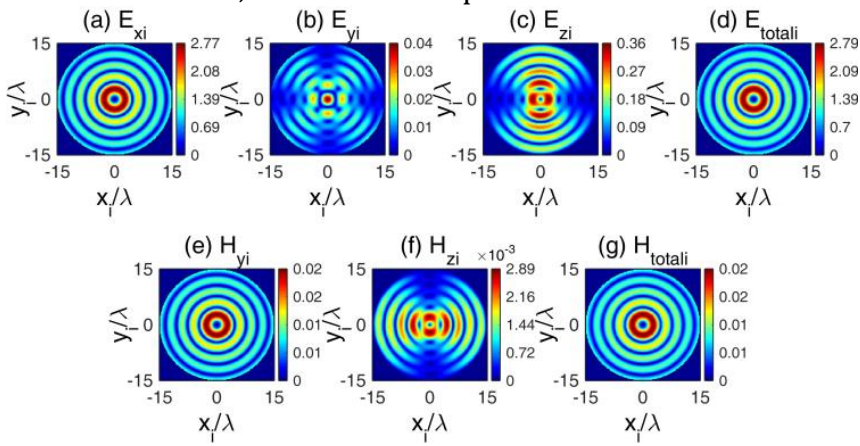

Fig.2. Magnitude distribution of the electric and the magnetic fields for a paraxial vector Bessel vortex beam $\left(\vartheta_{0}=10^{\circ}\right)$. The unit of magnitude is arbitrary.
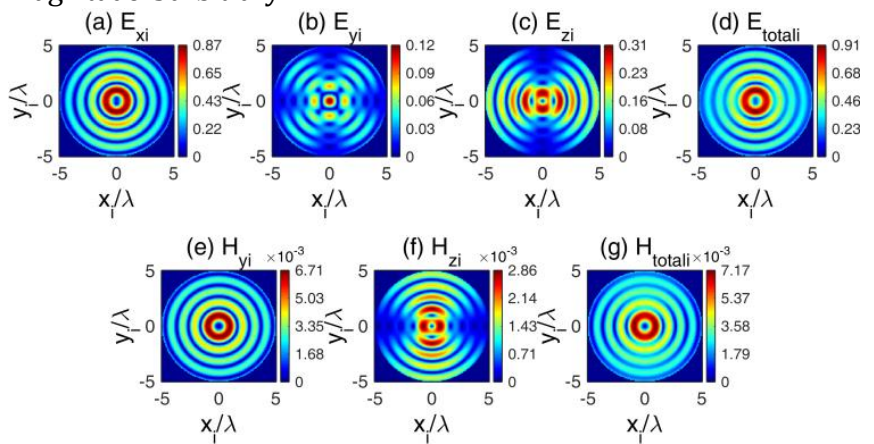

Fig.3. Magnitude distribution of the electric and magnetic fields for a non-paraxial vector Bessel vortex beam $\left(\vartheta_{0}=30^{\circ}\right)$. The unit of magnitude is arbitrary.

As shown in Fig.2, for the paraxial case, the distribution of field components $E_{x i}$ and $H_{y i}$ is circularly symmetric, while the other field components are axisymmetric about the $x_{i}$ and the $y_{i}$ axes, and the longitudinal field components $E_{z i}$ and $H_{z i}$ have the similar profiles but shifted by $90^{\circ}$. Because the field components $E_{x i}$ and $H_{y i}$ dominate, the profiles of the total electric and magnetic fields $E_{\text {totali }}$ and $H_{\text {totali }}$ are circularly symmetric. The profiles of field components in Fig.2 are similar to the results of Ref. [24], but the field component $E_{y i}$ is replaced by $H_{x i}$. However, for the non-paraxial case illustrated in Figs.3, all field components are axisymmetric except for the field component $H_{y i}$ which is circularly symmetric. The profiles of $E_{\text {totali }}$ and $H_{\text {totali }}$ are also axisymmetric due to the dominant field components $E_{z i}$ and $H_{z i}$.

\section{REFLECTED AND TRANSMITTED FIELDS FROM MULTILAYERED ISOTROPIC MEDIA USING VECTOR ANGULAR SPECTRUM METHOD}

Based on the vector angular spectrum expansion method, the electric field amplitudes of the reflected and transmitted Bessel vortex beams can be obtained by inverse Fourier transformation as:

$$
\begin{aligned}
\vec{E}_{r}= & \frac{e^{i k_{z r} z_{r}}}{2 \pi} \sum_{\lambda=1}^{2} \int \hat{e}_{\lambda}\left(k_{x r}, k_{y r}, \theta_{r}\right) \bar{R}_{\alpha} \tilde{A}_{\lambda}\left(k_{x i}, k_{y i}\right) . \\
& \quad \exp \left(i k_{0} r_{r} \sin \vartheta \cos \left(\phi_{r}-\varphi_{r}\right)\right) \sin \vartheta_{r} \cos \vartheta_{r} d \vartheta_{r} d \phi_{r}, \\
\vec{E}_{t}= & \frac{e^{i k_{z t} z_{t}}}{2 \pi} \sum_{\lambda=1}^{2} \int \hat{e}_{\lambda}\left(k_{x t}, k_{y t}, \theta_{t}\right) \bar{T}_{\alpha} \tilde{A}_{\lambda}\left(k_{x i}, k_{y i}\right) . \\
& \quad \exp \left(i k_{0} r_{t} \sin \vartheta \cos \left(\phi_{t}-\varphi_{t}\right)\right) \sin \vartheta_{t} \cos \vartheta_{t} d \vartheta_{t} d \phi_{t},
\end{aligned}
$$

where $\bar{R}_{\alpha}, \bar{T}_{\alpha}(\lambda=1, \alpha=p ; \lambda=2, \alpha=s)$ are the generalized reflection and transmission coefficients for both parallel and vertical polarizations, respectively [49]. Their expressions are presented in Appendix.

From Sell's law, considering the phase-matching conditions in the interface, we can express the relationship of transverse wave vector components between the incident wave and the reflected wave, the transmitted wave as $k_{x i}=k_{x r}, k_{y i}=-k_{y r}, \phi_{r}=-\phi_{i} \quad$ and $k_{x t}=k_{x i} / \eta, k_{y t}=k_{y i}, \phi_{t}=\arctan \left(k_{y t} / k_{x t}\right), \eta=\cos \theta_{t} / \cos \theta_{i}$, respectively. Thus, the polarization unit vectors in reflected coordinates and transmitted coordinates are written as

$\hat{e}_{1}\left(\vec{k}_{r t}\right)=\hat{e}_{1}\left(k_{x i},-k_{y i},-\theta\right), \hat{e}_{2}\left(\vec{k}_{r t}\right)=\hat{e}_{2}\left(k_{x i},-k_{y i},-\theta\right)$ and $\hat{e}_{1}\left(\vec{k}_{t t}\right)=\hat{e}_{1}\left(k_{x i} / \eta, k_{y i}, \theta_{t}\right), \hat{e}_{2}\left(\vec{k}_{t t}\right)=\hat{e}_{2}\left(k_{x i} / \eta, k_{y i}, \theta_{t}\right)$.

Considering the relationship between electric field and magnetic field shown in Eq.(4), the complex magnetic field magnitudes of the reflected and the transmitted Bessel beams also have the similar expressions as Eq.(6) and Eq.(7).

It is known that the intensity of electromagnetic fields is related to the time-averaged Poynting vector power density by $I \propto\langle\vec{S}\rangle \cdot \hat{e}_{z \beta}$, where the magnitude $\langle\vec{S}\rangle$ is given by:

$$
\left(\begin{array}{l}
\left\langle S_{x \beta}\right\rangle \\
\left\langle S_{y \beta}\right\rangle \\
\left\langle S_{z \beta}\right\rangle
\end{array}\right)=\frac{1}{2} \operatorname{Re}\left(\begin{array}{l}
E_{y \beta} \cdot H_{z \beta}^{*}-E_{z \beta} \cdot H_{y \beta}^{*} \\
E_{z \beta} \cdot H_{x \beta}^{*}-E_{x \beta} \cdot H_{z \beta}^{*} \\
E_{x \beta} \cdot H_{y \beta}^{*}-E_{y \beta} \cdot H_{x \beta}^{*}
\end{array}\right) .
$$

It is sufficient to consider the magnitude of $\langle\vec{S}\rangle$ for analyzing the characteristics of intensity distribution. Since the angular spectrum of the scalar Bessel beam has a Dirac function term about the half-cone angle $\vartheta_{0}$, the expressions in Eq.(6) and Eq.(7) can be conveniently reduced to one-dimensional integrals about the angle $\phi_{r, t}$ within the range of $[0,2 \pi]$. The results of the field components in Eq.(6) and Eq.(7) can be evaluated with the numerical integration method.

In order to analyze the effects of the media on polarization, similar to the definite of PER (polarization extinction ratio), the following expression is used to describe the polarization characteristics of the reflected and transmitted beam:

$$
P o l=10 \lg \frac{\iint S_{z r(t), x} d x_{r(t)} d y_{r(t)}}{\iint S_{z r(t), y} d x_{r(t)} d y_{r(t)}},
$$

where $S_{z r(t), x}$ denotes the time-averaged Poynting vector power density caused by the main component of the reflected or transmitted beam $E_{x r(t)}$, and $S_{z r(t), y}$ means the corresponding element for the $y$ components.

Meanwhile, in order to view variation of the longitudinal fields on the propagation of Bessel vortex beam, a similar factor $\mathrm{Pol}_{z}$ is defined and written as: 


$$
\operatorname{Pol}_{z}=10 \lg \frac{\iint S_{z r(t), x} d x_{r(t)} d y_{r(t)}}{\iint S_{t r(t), z} d x_{r(t)} d y_{r(t)}} .
$$

\section{NUMERICAL SIMULATION AND DISCUSSION}

\section{A. Verification of the method}

As we know, Bessel vortex beam is made up of the coherent superposition of a number of plane wavelets with same amplitude, and their wave vectors have different azimuth angles, but they have equal angles with the $z_{i}$ axis. If we set $n=0$ and $\vartheta_{0}=0^{\circ}$, the beam degrades to plane wave. Thus, in order to verify the correctness of the method provided in the paper, the reflection and transmission of zeroorder Bessel beam and plane wave incident on a dielectric interface are studied by it. The refractive indexes on both sides of the interface are set to $n_{1}=1.0$ and $n_{2}=1.38$, respectively.

To better understand the physical process of the propagation Bessel beam, the reflection and transmission coefficients for one interface case are plotted in Fig.4 and Fig.5. Each figure contains four subgraphs, which are corresponding with the reflection and transmission coefficients $R_{p}, T_{p} R_{s}$, and $T_{s}$, respectively. The subscripts $p$ and $s$ are for p-polarization and s-polarization. The horizontal axis denotes the azimuth of the plane wavelet in the $\boldsymbol{k}$ domain.
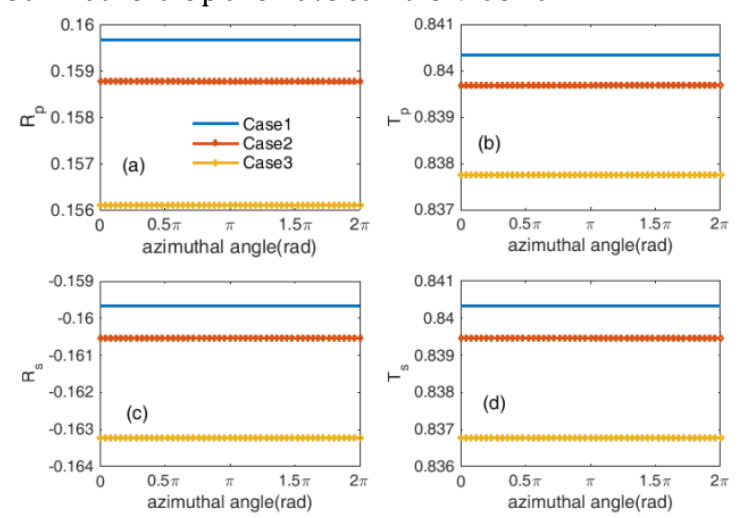

Fig.4. Reflection and transmission coefficients of one interface for the incident angle $\theta=0^{\circ}$.
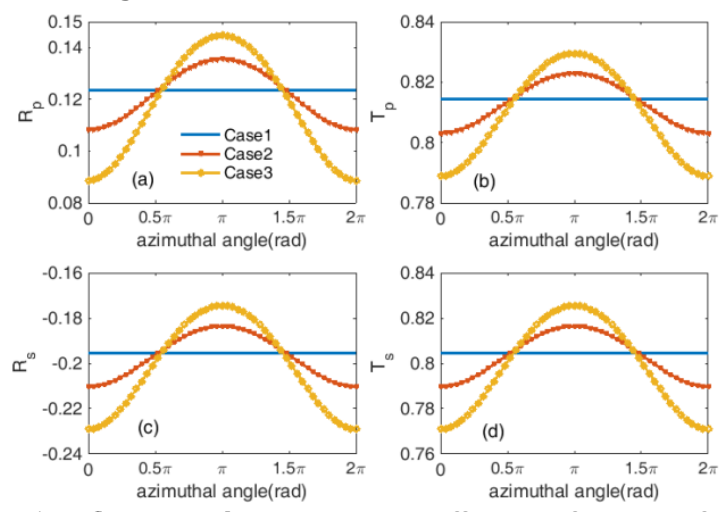

Fig.5. Reflection and transmission coefficients of one interface for the incident angle $\theta=30^{\circ}$.

In Figs. 4 and 5, case1, case 2 and case 3 are corresponding to the halfcone angles $\vartheta_{0}=0.0001^{\circ}, \vartheta_{0}=5^{\circ}$ and $\vartheta_{0}=10^{\circ}$, respectively. It is revealed that all the reflection and transmission coefficients for the half-cone angle $\vartheta_{0}=0.0001^{\circ}$ didn't change with the azimuthal angle, because for this case, beam is approximated to a plane wave. Meanwhile, the values of these coefficients are well coincided with the results directly from Fresnel coefficients. It is illustrated in Fig.4 that all the coefficients are constant with the azimuthal angle, which is due to all plane wavelets have the same incident angle (just equals the halfcone angle). However, when the incident angle is $\theta=30^{\circ}$, Fig.5 reveals that the reflection and transmission coefficients change with the azimuthal angle for the bigger half-cone angles and for different azimuthal angles, which is due to plane wavelets have different incident angles in this situation.

It is interesting that, for the vertical incidence, if we set the half-cone angle equals Brewster's angle $\vartheta_{0}=\theta=54.0715^{\circ}$, the influence of Brewster's law on the reflection of Bessel vortex beam can be analyzed.
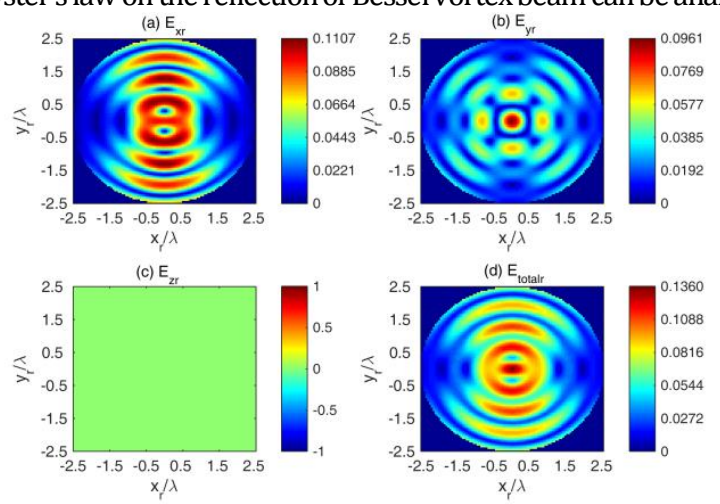

Fig.6. Distribution of Reflected electric fields as the incident angle is 0 degree, and the half-cone angle equals Brewster's angle.

The reflected electric fields are displayed in Fig.6. Unlike the results of a plane wave incident with the Brewster's angle, the $p$-polarization vanishes; the reflected electric field $\left|E_{x r}\right|$ doesn't disappear. For each plane wavelet, in the corresponding incident plane, the incident angle is just the Brewster's angle, and the $p$-polarization of its reflected field disappears. Whereas, this phenomenon is only effective in that incident plane, not the plane of the paper. However, when we consider the $x_{i}$ polarization of Bessel vortex beam, the total result of the reflected field component $\left|E_{x r}\right|$, which is the superposition of all plane wavelets in the plane $x_{r} o y_{r}$, doesn't vanish.

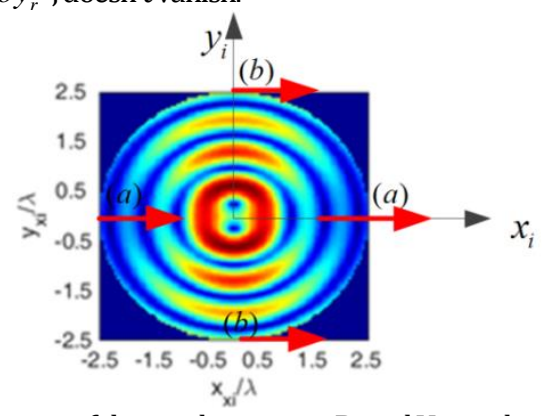

Fig.7. Polarization of the incident vector Bessel Vortex beam, (a) and (b) are two special locations.

A schematic diagram of the incident beam on the plane $x_{i} o y_{i}$ drawn in Fig.7 is used to explain the above phenomenon in more detail. 
Considering the relationship between local coordinate systems $\left(x_{i}, y_{i}, z_{i}\right)$ and $\left(x_{r}, y_{r}, z_{r}\right)$, the $p$-polarization of plane wavelets in the plane $x_{i} o z_{i}$ will vanish through reflection and has no contribution on the electric field $\left|E_{x r}\right|$, that is the case(a) in Fig.7, whereas, for other azimuthal angles case, they all have contribution on the distribution of $\left|E_{x r}\right|$. For example, the $p$-polarization of plane wavelets in the plane $x_{i} o y_{i}$ (case(b) in Fig.7) also will disappear, but its s-polarization is just along the $x_{r}$ axis, that is to say, it makes great contribution to the electric field $\left|E_{x r}\right|$.

\section{B. Magnitude distribution of the reflected and transmitted fields} of three-layered media for the paraxial case

Three-layered media are considered whose layers' refractive indices and thicknesses are shown in Table1. The materials selected here are often used to produce optical thin film. The wavelength and the topological charge of the incident beam are considered to be $\lambda_{0}=0.5 \mu \mathrm{m}$ and $n=2$, respectively. The polarization mode is the same to that used in Figs.2, 3.

Table1. Refractive indices and thicknesses of the three-layered media.

\begin{tabular}{|l|c|c|}
\hline Layer/Name of material & Refractive index & thickness \\
\hline 1/Silicon dioxide & 1.38 & $5 \mu \mathrm{m}$ \\
\hline 2/Aluminium Oxide & 1.63 & $5 \mu \mathrm{m}$ \\
\hline 3/Titanium Sesquioxide & 2.35 & $5 \mu \mathrm{m}$ \\
\hline
\end{tabular}

For a paraxial Bessel beam, the half-cone angle is set to be $\vartheta_{0}=10^{\circ}$, and the incident angle is $\theta=30^{\circ}$. Figs.8, 9 display the magnitude distributions of both reflected and transmitted field components, and the unit of magnitude is arbitrary. It can be seen that all the reflected and the transmitted beams consist of six field components. The magnitudes of the transverse field components are still dominant in the total field components.

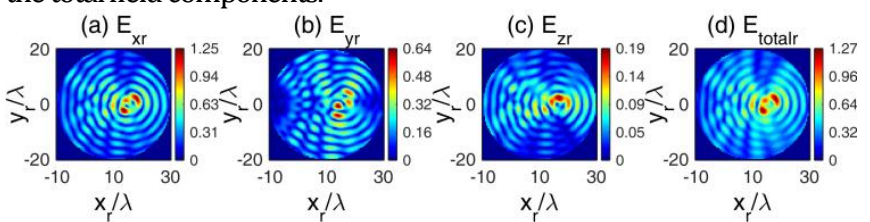

(e) $\mathrm{H}_{\mathrm{xr} \times 10^{3}}$

(h) $\mathrm{H}$

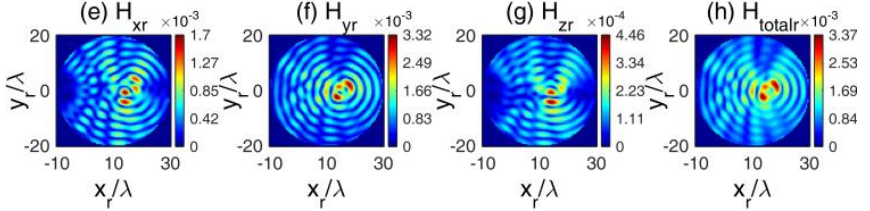

Fig.8. Magnitude distribution of the reflected electric and magnetic fields from three-layered media.
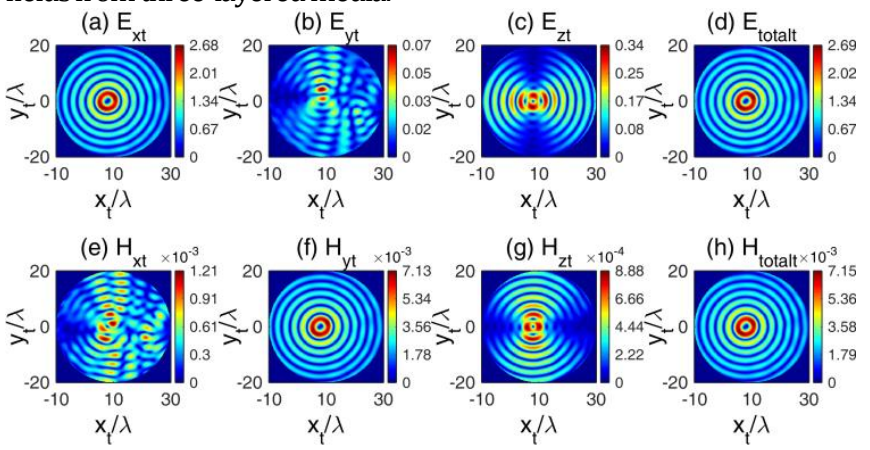

(h) $\mathrm{H}_{\text {totalt } \times 10^{-3}}$

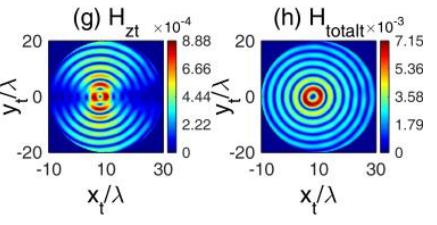

Fig.9. Magnitude distribution of the transmitted electric and magnetic fields from three-layered media.

In the reflected beam case shown in Fig.8, the magnitude distributions of all the field components are no longer circularly symmetric or axisymmetric due to the effects of the multiple reflection and transmission in the media. Compared with the distributions of the incident field components plotted in Fig.2, the distortions of the magnitude profiles are very notable. The profiles of each field component represent the overall reflection from the four interfaces of the three-layered media. It can be seen that the field components $E_{x r}$ and $H_{y r}$, as well as the field components $E_{y r}$ and $H_{x r}$ have similar distribution patterns, while the magnitude profiles of the field components $E_{z r}$ and $H_{z r}$ also have similar distribution except for a $90^{\circ}$ phase shift.

As shown in Fig.9, for the transmitted beam, except the field components $E_{y t}$ and $H_{x t}$, the magnitude profiles of other field components have distribution patterns similar to those of the incident field components in Fig.2, but the profiles of the field components $E_{x t}$ and $H_{y t}$ are no longer perfectly circularly symmetric.

\section{Magnitude distribution of the reflected and transmitted fields for the non-paraxial case}

Figs.10, 11 show the magnitude profiles of the reflected and the transmitted field components for the non-paraxial case with a halfcone angle $\vartheta_{0}=30^{\circ}$. The incident angle is $\theta=20^{\circ}$.
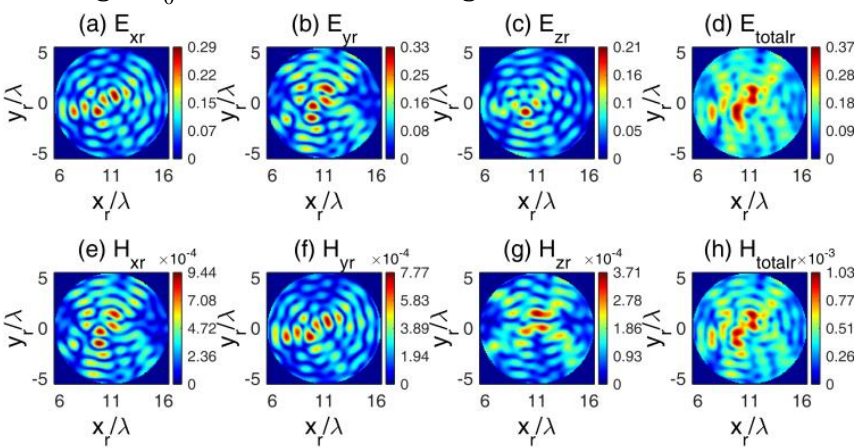

Fig.10. Magnitude distribution of the reflected electric and magnetic fields from three-layered media.
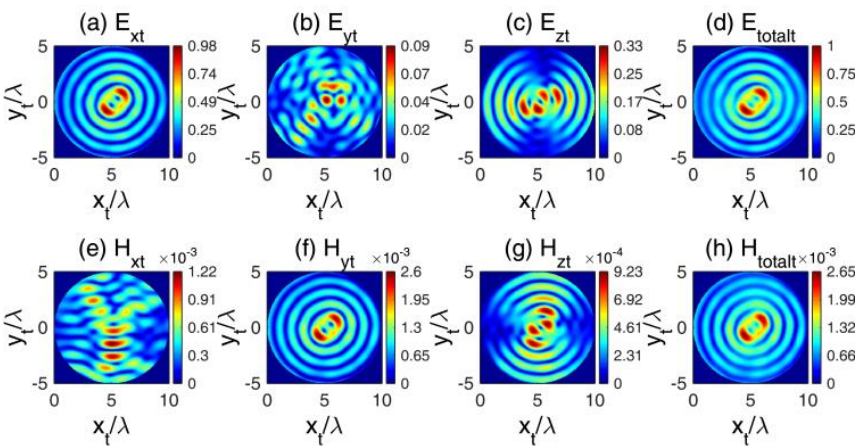

Fig.11. Magnitude distribution of the transmitted electric and magnetic fields from three-layered media.

Compared with the results of the paraxial case in Figs.8, 9, the magnitude distributions of reflected field components in Fig.10 are more distorted than those of the paraxial case. Unlike the paraxial case, the magnitudes of the longitudinal field components $E_{z r}$ and $H_{z r}$ 
also play important roles in those of the total field components $E_{\text {totalr }}$ and $H_{\text {totalr }}$. For the transmitted beam, although the ratios of the longitudinal field components $E_{z t}$ and $H_{z t}$ to the corresponding transverse field components $E_{x t}$ and $H_{y t}$ are bigger than the paraxial case shown in Fig.9, the magnitudes of the transverse components $E_{x t}$ and $H_{y t}$ are still dominant in the total field components. Meanwhile, the magnitudes of the centers of the field components $E_{x t}$ and $H_{y t}$ are no longer equal to zero.

From the distribution profiles in Figs.8-11, it can be observed that the magnitude centroids of all the field components of both reflected and transmitted beam have notable offsets in the positive directions of the $x_{\gamma}(\gamma=r, t)$ axes, which are mostly influenced by both the thicknesses and the refraction indices of the media, as well as the incident angle of the Bessel vortex beam. Nevertheless, the offsets are not evident in the directions of the $y_{\gamma}(\gamma=r, t)$ axes. The offsets of the reflected beam are bigger than those of the transmitted beam.

Based on Snell's law, the offsets of one ray in the $x_{\gamma}(\gamma=r, t)$ axes are illustrated by Fig.12.

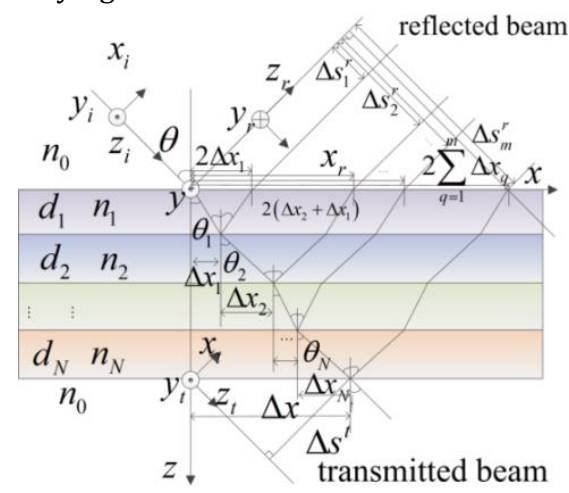

Fig.12. Schematic of reflected and transmitted rays in multilayered isotropic media.

When only the primary transmission is considering, the offset canbe obtained as

$$
\Delta s^{t}=\Delta x \cos \theta
$$

where $\Delta x=\sum \Delta x_{q}$, The movement of beam along positive $x_{t}$ axis in each layer $\Delta x_{q}$ can be obtained through the Snell's law.

Substituding the paramenters in Fig.9 into Eq.(11), the offset of transmitted field can be obtained, and its value is $7.952 \lambda$, which is well coincided with the numerical result in Fig.9.

In order to clearly view the intensity characteristics of the Bessel vortex beam through the three-layered media, the magnitude distributions of $\langle\vec{S}\rangle$ for the incident, the reflected and the transmitted beams are also simulated in next two sections.

\section{Magnitude distribution of $\langle\vec{S}\rangle$ for the paraxial case}

For the paraxial case, three cases for the incident angles $0^{\circ}, 20^{\circ}$ and $30^{\circ}$ are plotted in Figs.13, 14. The first to third lines are the transverse, the longitudinal and the total time-averaged Poynting vector power densities $\left\langle S_{t}\right\rangle,\left\langle S_{z}\right\rangle$ and $\left\langle S_{\text {total }}\right\rangle$, respectively.

For the reflected beam case presented in Fig.13, the maximum values of the transverse components $\left\langle S_{t}\right\rangle$ are ten times smaller than those of the longitudinal components $\left\langle S_{z}\right\rangle$. The magnitudes of $\left\langle S_{\text {total }}\right\rangle$ are mainly decided by the longitudinal components $\left\langle S_{z}\right\rangle$. The magnitude profiles of the longitudinal and the total components for the incident angle $\theta=0^{\circ}$ almost have the same circularly symmetric distribution patterns to those of the incident beam, whereas a small change in the profile of the transverse component $\left\langle S_{t}\right\rangle$ is observed. As the incident angle increases from $\theta=0^{\circ}$ to $\theta=30^{\circ}$, the distortions of the magnitude profiles for all components are becoming more significant, but can still see the multiple concentric rings.

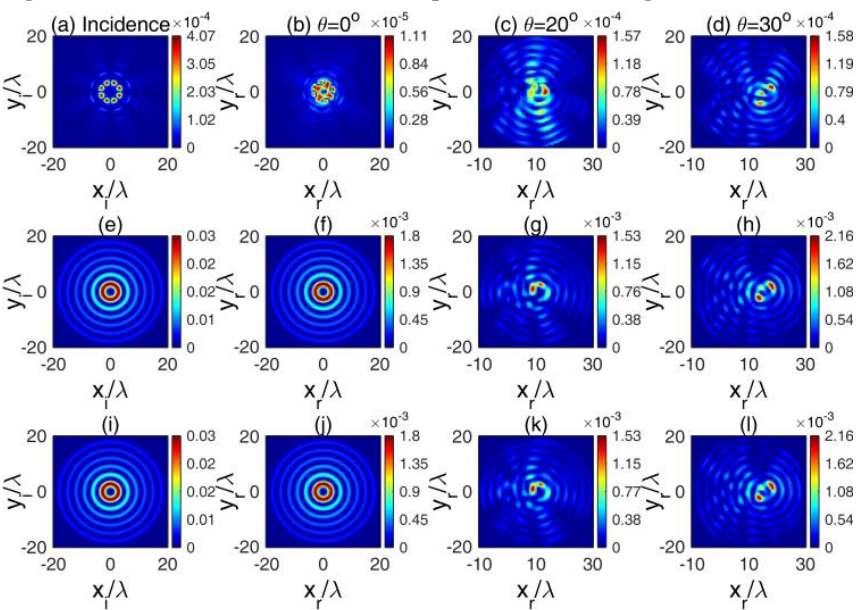

Fig.13 Variations of the magnitude profiles of the reflected beam components of the time-averaged Poynting vector with the incident angle for the paraxial case.
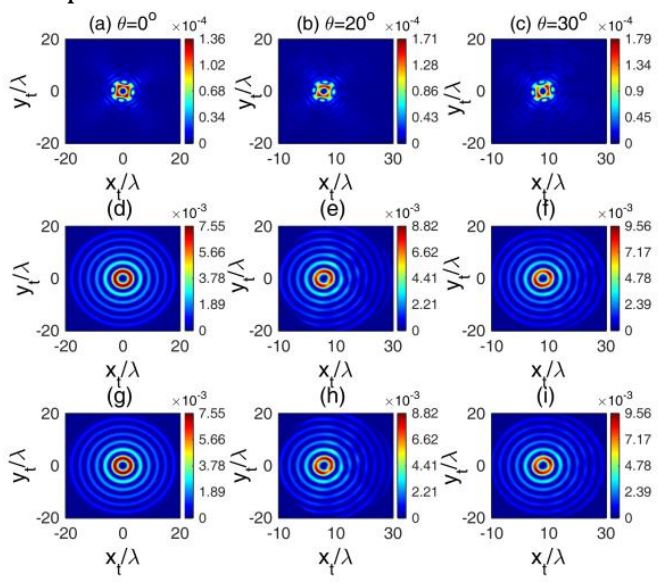

Fig.14. Variations of the magnitude distributions of the transmitted beam components of the time-averaged Poynting vector with the incident angle for the paraxial case.

For the transmitted beam displayed in Fig.14, except for the transverse components, other components for the incident angle $\theta=0^{\circ}$ have the same distribution patterns to those of the incident beam. As the incident angle increases, the profile of the transverse component is almost unchanged, but the magnitude distributions of the longitudinal and the total components are gradually deviating from the circular symmetry, especially their central rings.

Although the reflected and the transmitted beams have all four transverse field components, which are different from the incident beam case, the magnitudes of field components $E_{x r}, H_{y r}$ and $E_{x t}, H_{y t}$ are still dominant in the longitudinal components. In other 
words, the distribution profiles of the longitudinal components for both the reflected and the transmitted beams, are similar to the corresponding transverse field components.

\section{E. Magnitude distribution of $\langle\vec{S}\rangle$ for the non-paraxial case}

For the non-paraxial case, three cases for the incident angles $0^{\circ}$, $10^{\circ}$ and $20^{\circ}$ are illustrated in Figs.15, 16. The parameters of the incident beam are the same to those in Fig 11.

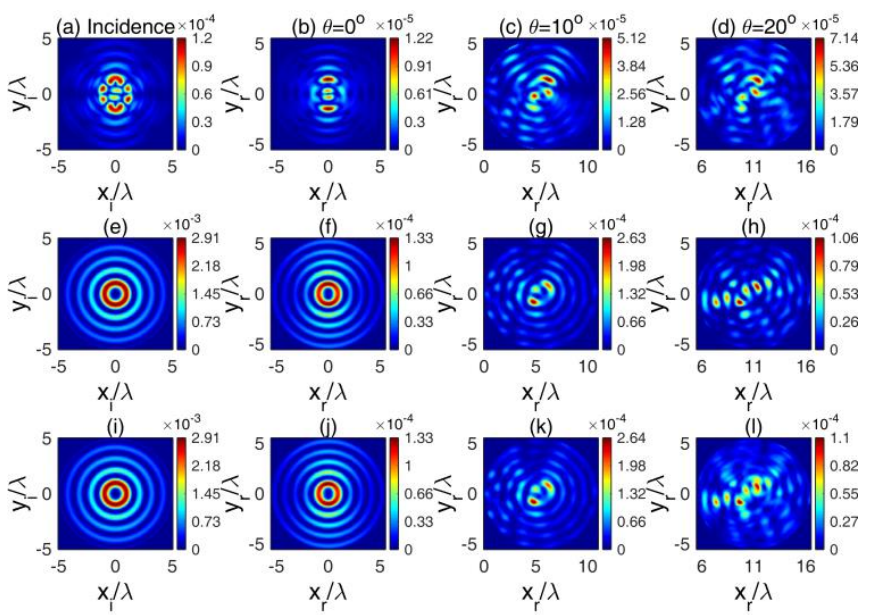

Fig.15. Variations of the magnitude profiles of the reflected beam components of the time-averaged Poynting vector with the incident angle for the non-paraxial case.

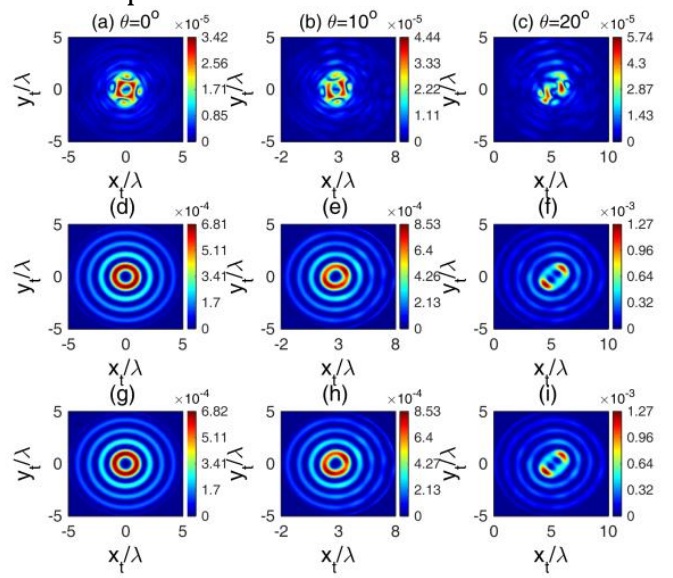

Fig.16. Variations of the magnitude distributions of the transmitted beam components of the time-averaged Poynting vector with the incident angle for the non-paraxial case.

For the reflected beam shown in Fig.15, the magnitude distributions of $\left\langle S_{t}\right\rangle,\left\langle S_{z}\right\rangle$ and $\left\langle S_{\text {total }}\right\rangle$ of the incident beam are no longer strictly circular, but they are still axisymmetric. When the incident angle is $\theta=0^{\circ}$, the magnitude distributions of the longitudinal and the total components have patterns similar to those of the incident beam, but they are more deviated from the circular symmetry. As the incident angle is increased to 10 degrees, the magnitude distributions of the longitudinal and the total components still appear to be multiple concentric rings. However, it is clear that as the incident angle increases further, the distortions of the magnitude distributions are more notable and cannot maintain the concentric ring structure. In the case of the same incident angle, the distortions of the magnitude distributions for the non-paraxial case are more notable, and the effects of $\left\langle S_{t}\right\rangle$ on $\left\langle S_{\text {total }}\right\rangle$ the magnitude distribution of are more significant than those of the paraxial case, which are given in Fig.13.

For the transmitted beam results shown in Fig.16, with an increase of the incident angle, the magnitude distributions of the longitudinal and the total components have more obvious changes than those of the paraxial case, especially the central ring, which is no longer circular. Meanwhile, for the same incident angle, the distortions of the central rings in the magnitude distributions illustrated in Fig.16(f) and Fig.16(i), are more evident than those of the paraxial case.

\section{F. Variation of phase for the main components $E_{x}$ of the reflected and transmitted fields}

Phase distribution can reflect the orbit angular momentum features of the vortex beams, a discussion about the effects of multilayered media on phase distribution is necessary.

For the vertical incidence of a paraxial beam, phase distributions for the main electric field components $E_{x \beta}(\beta=i, r, t)$ in the planes $x_{\beta}-y_{\beta}$ are shown in Fig.17. Obviously, the phase contour of the incident beam in Fig.17(a) consists of multiple concentric rings, just coincides with the corresponding magnitude distribution in Fig.2(a), and the phase value on each ring varies from 0 to $2 \pi$ for two times. The results in Fig.17 (b) and (c) show that the phase distributions of the reflected and the transmitted fields $E_{x \gamma}(\gamma=r, t)$ remain similar to that of the incident beam
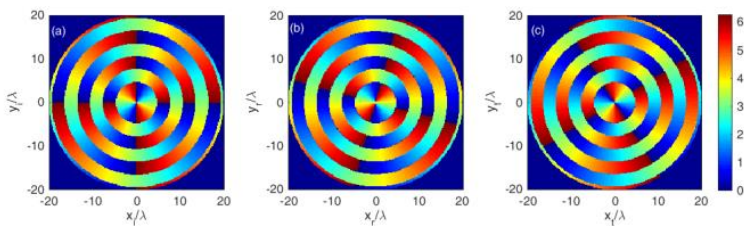

Fig.17. Phase distributions for field components $E_{x \beta}(\beta=i, r, t)$ as a paraxial vector Bessel vortex beam incident vertically on the threelayered media, (a)incident beam, (b) reflected field, (c)transmitted field.
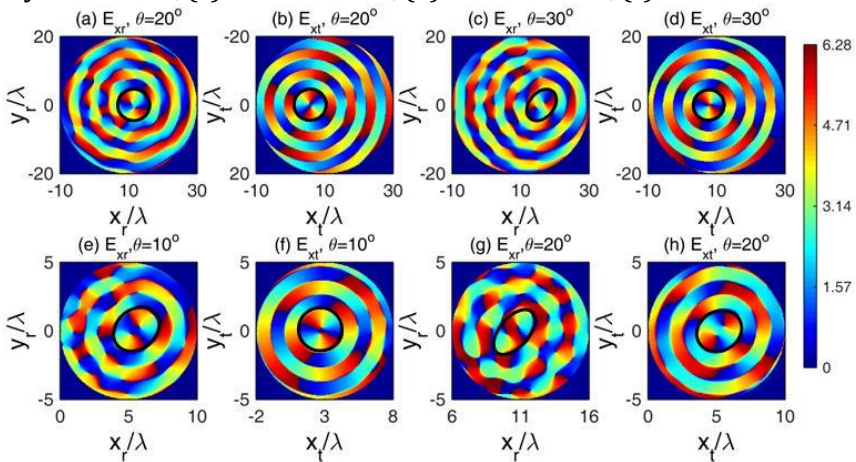

Fig.18. Phase distributions for the main electric components as a vector Bessel vortex beam irradiates on the media with different incident angles. Panels (a)-(d) are the results for paraxial case, while (e)-(h) for those of non-paraxial case.

The effects of the incident angle on phase distribution are illustrated in Fig.18 Panels in the first row are results for the paraxial case, and others are for the non-paraxial case. It is concluded from Fig.18 that distortions of the phase contours for the reflected fields are more pronounced than that for the transmitted fields, and distortions for all 
fields become more significant as the increase of the incident angle. Whereas the phases on the innermost ring in Fig.18(a), (c), (e) and (g) are basically able to maintain the shape similar to the incident fields. Phase distributions of the transmitted fields almost keep the concentric rings, even though the innermost ring gradually becomes elliptical as the incident angle increases. It should be noted that for most of the reflected and transmitted fields, compared to the incident beam, the points of the center phase singularities or become larger, or disappear. For the same incident angle, the distortion of phase distribution for the non-paraxial case are more serious than that for the paraxial case.

\section{G. Pol and Polz of the reflected and transmitted fields}

Using the definition in Eqs.(9) and (10), the values of Pol and Polz for both the paraxial case and the nonparaxial case are calculated and shown in Table2 and Table3.

As we know the polarization unit vector of the incient beam is $\hat{f}=\hat{x}_{i}$, in other words, the electric field $E_{x i}$ is the main component. From Table2, after the interaction between the beam and the media, the components $E_{x \gamma}$ are still prominent for the incident angle $\theta=0^{\circ}$. However, as the incident angle changes, the polarization components $E_{y r} E_{z r}$ increase significantly, whereas the polarization components $E_{y t}, E_{z t}$ increase slowly.

Table2. Pol and Polz of the reflected and transmitted fields for paraxial case (Unit:dB)

\begin{tabular}{|l|l|l|l|}
\hline $\begin{array}{l}\text { Incidentangle } \\
\text { Four cases }\end{array}$ & $\theta=0^{\circ}$ & $\theta=20^{\circ}$ & $\theta=30^{\circ}$ \\
\hline Pol (Reflected fileds) & 35.96 & 0.433 & 5.96 \\
\hline Pol (Transmitted fileds) & 47.19 & 39.18 & 35.39 \\
\hline Polz (Reflected fileds) & 25.04 & 7.9422 & 10.93 \\
\hline Polz (transmitted fileds) & 21.86 & 21.45 & 21.31 \\
\hline
\end{tabular}

For the non-paraxial case, all values of Pol and Polz are smaller than the results of the paraxial case. For the incident angle $\theta=20^{\circ}$,the value Pol(Reflected fields) is -1.92 , which shows that the polarization component $E_{y r}$ predominate, besides, the Polz(Reflected fields) is also very small, which illustrates the polarization component $E_{z r}$ increases.

Table3. Pol and Polz of the reflected and transmitted fileds for the nonparaxial case (Unit:dB)

\begin{tabular}{|c|c|c|c|}
\hline $\begin{array}{l}\text { Incident angle } \\
\text { Four cases }\end{array}$ & $\theta=0^{\circ}$ & $\theta=10^{\circ}$ & $\theta=20^{\circ}$ \\
\hline Pol (Reflected fileds) & 15.61 & 6.31 & -1.92 \\
\hline Pol (Transmitted fileds) & 27.25 & 30.78 & 33.03 \\
\hline Polz(Reflected fileds) & 12.57 & 6.21 & 1.15 \\
\hline Polz (transmitted fileds) & 15.12 & 14.75 & 14.51 \\
\hline
\end{tabular}

\section{Conclusions}

Reflection and transmission characteristics of a Bessel vortex beam from multilayered media are studied on the basis of the vector angular spectrum expansion and the generalized reflection and transmission coefficients. The magnitudes of the field components of a linearly polarized Bessel vortex beam are calculated for both paraxial and nonparaxial cases. It is shown that the magnitude distributions of the total field components for the paraxial case are circularly symmetric, and are mainly dominated by the magnitudes of the transverse field components, but as the half-cone angle increases, the effects of other field components on the total field components, especially the longitudinal field components cannot be neglected.

The reflected and the transmitted electric fields are expressed using the inverse Fourier transform integrals of the vector angular spectrum in the spherical coordinate system and are solved by the numerical method. Through studying the reflection and transmission on a single interface, the method is verified. When the half-cone angle is equal to the Brewster's angle at the vertical incidence, the reflectied field components are displayed and discussed. For the incident Bessel vortex beams with the half-cone angles $\vartheta_{0}=10^{\circ}$ and $\vartheta_{0}=30^{\circ}$, the magnitude distributions of the field components, the time-averaged Poynting vector power density, the phases and the polarization from three-layered media are analyzed in detail. The results reveal that the distortions of magnitude distributions of the reflected field components are very notable, but the magnitude distributions of the transmitted field components can still keep the similar patterns to the corresponding incident beams to some extent. As the incident angle increases, variations of magnitude distributions of the time-averaged Poynting vector power density for the reflected beam become significant, the total components for the paraxial case are still mainly determined by the longitudinal components, while for the non-paraxial case, the effects of the transverse components on the magnitude distribution of the total components must be taken into consideration. The magnitude distributions of the field components, and the timeaveraged Poynting vector power density in their local coordinates all have evident offsets in the positive directions of the $x_{\gamma}(\gamma=r, t)$ axes, which is greatly influenced by both the thicknesses and the refractive indices of the media, as well as the incident angle of the vortex Bessel beam. The distortion of the phase distributions for reflected fields is more serious, and most phase singularies or vanish or increase. Compared to the incident beam, the polariaztion of the reflected beam changed seriously. The results of this paper may provide some suggestion for the vortex signal receiving after the interaction between vortex beam and media.

The method presented in this work can be used to analyse the propagation characteristics of vector Bessel vortex beams with arbitrary polarization mode in isotropic inhomogeneous media, which may promote the tremendous potential application of the Bessel vortex beam in many fields, such as vortex beam communication, design of optical devices and optical thin films.

\section{Appendix A}

The polarization unit basis vectors are

$$
\hat{e}_{1}\left(\vec{k}_{i t}\right)=\frac{\left\{\begin{array}{l}
{\left[U W c+\left(W^{2}+V^{2}\right) s\right] \hat{x}_{i}+V(W c-U s) \hat{y}_{i}} \\
-\left[\left(U^{2}+V^{2}\right) c+U W s\right] \hat{z}_{i}
\end{array}\right\}}{\left\{\begin{array}{l}
{\left[U W c+\left(W^{2}+V^{2}\right) s\right]^{2}+V^{2}(W c-U s)^{2}} \\
+\left[\left(U^{2}+V^{2}\right) c+U W s\right]^{2}
\end{array}\right\}}
$$

Here $U, V$ and $W$ denote $k_{i x}, k_{i y}, k_{i z}$, respectively. The shorthands $c=\cos \theta$ and $s=\sin \theta$ are used, and $\theta$ is defined as $\theta=\arccos \left(\hat{z}_{i} \cdot \hat{z}\right)$.

In the description of the reflected and transmitted fields, the $\bar{R}_{\alpha}, \bar{T}_{\alpha}$ are needed. The recursive form $\bar{R}_{\alpha}$ of N-layers media is shown as:

$$
\bar{R}_{j, j+1}=\frac{R_{j, j+1}+\bar{R}_{j+1, j+2} \exp \left(i 2 k_{j+1, z}\left(d_{j+1}-d_{j}\right)\right)}{1+R_{j, j+1} \bar{R}_{j+1, j+2} \exp \left(i 2 k_{j+1, z}\left(d_{j+1}-d_{j}\right)\right)} .
$$




$$
\bar{T}_{\alpha}=\prod_{j=0}^{N} \exp \left(i k_{j, z}\left(d_{j}-d_{j-1}\right)\right) S_{j, j+1},
$$

where

$$
S_{j, j+1}=T_{j, j+1} /\left[1-R_{j+1, j} \bar{R}_{j+1, j+2} \exp \left(i 2 k_{j+1, z}\left(d_{j+1}-d_{j}\right)\right] .\right.
$$

The $R_{j+1, j}$ and $T_{j, j+1}$ in Eqs. (A-2) and (A-3) are the Fresnel reflection and transmission coefficients, respectively, and their detailed expressions for both parallel and vertical polarizations can be found in [49].

Funding Information. National Science Foundation (NSF) (61475123, 61571355, 61501350); Natural Science Basic Research Plan in Shaanxi Province of China(2016JQ4015); the 111 Project (B17035).

Acknowledgment. Farideh Honary acknowledges fruitful discussions of the results from this research with colleagues at the International Space Science Institute, Bern, Switzerland.

\section{References}

1. J. Durnin, "Exact solutions for non-diffracting beams. I. The scalar theory," J. Opt. Soc. Am. A. 4, 651 (1987).

2. J. Turunen, A. Vasara, and A. T. Friberg, "Holographic generation of diffraction-free beams," Appl. Opt. 27, 3959 (1988).

3. R. M. Herman and T. A. Wiggins, "Production and uses of diffractionless beams," J. Opt. Soc. Am. A. 8, 932 (1991).

4. J. Arlt and K. Dholakia, "Generation of high-order Bessel beams by use of an axicon," Opt. Commun. 177, 297 (2000).

5. H. Meng, B. Xiang, J. Zhang, W. Dou, and Y. Yu, "The Generation of Bessel Beam and Its Application in Millimeter Wave Imaging," J INFRARED MILLIM TE. 35, 208 (2014).

6. R. Bowman, N. Muller, X. Zambrana-Puyalto, O. Jedrkiewicz, P. D. Trapani, and M. J. Padgett, "Efficient generation of Bessel beam arrays by means of an SLM," Eur. Phys. J. 199, 156 (2011).

7. K. B. Kuntz, B. Braverman, S. H. Youn, M. Lobino, E. M. Pessina, and A. I. Lvovsky, "Spatial and temporal characterization of a Bessel beam produced using a conical mirror," Phys. Rev. A. 79, (2009).

8. Lei Gong, Yu-Xuan Ren, Guo-Sheng Xue, Qian-Chang Wang, Jin-Hua Zhou, Min-Cheng Zhong, Zi-Qiang Wang, and Yin-Mei Li, "Generation of nondiffracting Bessel beam using digital micromirror device," Appl. Opt. 52, 4566 (2013).

9. V. Garces-Chavez, D. McGloin, H. Melville, W. Sibbett, and K. Dholakia, "Simultaneous micromanipulation in multiple planes using a selfreconstructing light beam," Nature. 419, 145 (2002).

10. D. Mugnai, "Bessel beams and signal propagation," Phys. Lett. A. 278, 6 (2000).

11. F. G. Mitri, R. X. Li, L. X. Guo, and C. Y. Ding, "Optical tractor Bessel polarized beams," J Quant Spectrosc Radiat Transf. 187, 97 (2017).

12. H. Meng, B. Xiang, J. Zhang, W. Dou, and Y. Yu, "The Generation of Bessel Beam and Its Application in Millimeter Wave Imaging," J INFRARED MILLIM TE. 35, 208 (2014).

13. V. Kollarova, T. Medrik, R. Celechovsky, Z. Bouchal, O. Wilfert, and Z. Kolka, "Application of nondiffracting beams to wireless optical communications," Proc. SPIE 6736, 67361C (2007)

14. A. E. Willner, J. Wang, and H. Huang, "A Different Angle on Light Communications," Science. 337, 655 (2012).

15. Y. Yan, G. Xie, M. P. J. Lavery, H. Huang, N. Ahmed, C. Bao, Yongxiong Ren, Yinwen Cao, Long Li, Zhe Zhao, Andreas F. Molisch, Moshe Tur, Miles J. Padgett and Alan E. Wilner, "High-capacity millimeter-wave communications with orbital angular momentum multiplexing," Nat. Commun. 5, 4876 (2014).

16. A. E. Willner, H. Huang, Y. Yan, Y. Ren, N. Ahmed, G. Xie, C. Bao, L. Li, Y. Cao, Z. Zhao, J. Wang, M. P. J. Lavery, M. Tur, S. Ramachandran, A. F.
Molisch, N. Ashrafi, and S. Ashrafi, "Optical communications using orbital angular momentum beams," Adv. Opt. Photon. 7, 66 (2015).

17. A. Dudley, T. Mhlanga, M. Lavery, A. McDonald, F. S. Roux, M. Padgett, and A. Forbes, "Efficient sorting of Bessel beams," Opt. Express. 21, 165 (2013).

18. C. Schulze, A. Dudley, R. Brüning, M. Duparré, and A. Forbes, "Measurement of the orbital angular momentum density of Bessel beams by projection into a Laguerre-Gaussian basis," Appl. Opt. 53, 5924 (2014).

19. Y. Yuan, T. Lei, S. Gao, and X. Yuan, "Parallel Detection of OAM States Carried by Coaxial Bessel Beams," IEEE PHOTONIC TECH L. 28, 315 (2016).

20. F. G. Mitri, "Vector wave analysis of an electromagnetic high-order Bessel vortex beam of fractional type $\alpha$," Opt. Lett. 36, 606 (2011).

21. M. Ornigotti and A. Aiello, "Radially and azimuthally polarized nonparaxial Bessel beams made simple," Opt. Express. 21, 15530 (2013).

22. M. Ornigotti and A. Aiello, "Generalized Bessel beams with two indices," Opt. Lett. 39, 5618 (2014).

23. Y. Wang, W. Dou, and H. Meng, "Vector analyses of linearly and circularly polarized Bessel beams using Hertz vector potentials," Opt. Lett. 22, 7821 (2014).

24. J. J. Wang, T. Wriedt, J. A. Lock, and L. Mädler, "General description of circularly symmetric Bessel beams of arbitrary order," J Quant Spectrosc Radiat Transf. 184, 218 (2016).

25. J. J. Wang, T. Wriedt, J. A. Lock, and Y. C. Jiao, "General description of transverse mode Bessel beams and construction of basis Bessel fields," J Quant Spectrosc Radiat Transf. 195, 8 (2017).

26. G. Gouesbet, B. Maheu, and G. Gréhan, "Light scattering from a sphere arbitrarily located in a Gaussian beam, using a Bromwich formulation," J. Opt. Soc. Am. A. 5, 1427 (1988).

27. K. Y. Bliokh and A. Aiello, "Goos-Hänchen and Imbert-Fedorov beam shifts: an overview," J. Opt. 15, 014001 (2013).

28. N. I. Petrov, "Reflection and transmission of strongly focused vector beams at a dielectric interface," Opt. Lett. 29, 421 (2004).

29. H. Okuda and H. Sasada, "Significant deformations and propagation variations of Laguerre-Gaussian beams reflected and transmitted at a dielectric interface," J. Opt. Soc. Am. A. 25, 881 (2008).

30. H. Y. Li, F. Honary, Z. S. Wu and L. Bai, "Reflection and transmission of Laguerre-Gaussian beams in a dielectric slab," J Quant Spectrosc Radiat Transf. 195, 35 (2017).

31. Richard W. Ziolkowski, "Pulsed and CW Gaussian beam interactions with double negative metamaterial slabs," Opt. Express. 11, 662 (2003).

32. M. J. Wang, H.Y. Zhang, G.S. Liu, Y.L. Li, and Q.F. Dong, "Reflection and transmission of Gaussian beam by a uniaxial anisotropic slab," Opt. Express. 22, 3705 (2014).

33. B. Yan, H. Zhang, and J. Zhang, "Reflection and transmission of Gaussian beam by a chiral slab," Appl. Phys. B. 122, 174 (2016).

34. A. Aiello, J. P. Woerdman, "Goos-Hänchen and Imbert-Fedorov shifts of a nondiffracting Bessel beam," Opt. Lett. 36, 543 (2011).

35. D. Mugnai, "passage of a Bessel beam through a classically forbidden region," Opt. Commun. 188, 17 (2001).

36. D. Mugnai, "Bessel beam through a dielectric slab at oblique incidence: the case of total reflection," Opt. Commun. 207, 95 (2002).

37. D. Mugnai, "Propagation of Bessel beams from a dielectric to a conducting medium," Appl. Opt. 50, 2654 (2011).

38. D. Mugnai and P. Spalla, "Electromagnetic propagation of Bessel-like localized waves in the presence of absorbing media," Opt. Commun. 282, 4668 (2009).

39. A. V. Novitsky and L. M. Barkovsky, "Total internal reflection of vector Bessel beams: Imbert-Fedorov shift and intensity transformation," J. Opt. A: Pure Appl. Opt. 10, 075006 (2008).

40. A. V. Novitsky, D. V. Novitsky, "Change of the size of vector Bessel beam rings under reflection," Opt. Commun. 281, 2727 (2008).

41. F. Falco and T. Tamir, "Improved analysis of nonspecular phenomena in beams reflected from stratified media," J. Opt. Soc. Am. A. 7, 185 (1990).

42. Y. Wang, Y. Liu, J. Xu, H. Zhang, L. Bai, Y. Xiao, J. Yan and X. Zhang, "Numerical study of lateral displacements of Gaussian beams reflected 
from weakly absorbing media near the Brewster dip and reflected from strongly absorbing media," J. Opt. A: Pure Appl. Opt. 11, 105701 (2009).

43. H. Mao, T. Zang, J. Sun, T. Pan, and G. Xu, "Goos-Hanchen shifts of the reflected waves from the inhomogeneous slab with a positive and negative index transition layer," Phys. Status Solidi B. 249, 829 (2012).

44. O. Manela, M. Segev, and D. N. Christodoulides, "Nondiffracting beams in periodic media," Opt. Lett. 30, 2611 (2005).

45. A. V. Novitsky and L. M. Barkovsky, "Vector Bessel beams in bianisotropic media," J. Opt. A: Pure Appl. Opt. 7, 550 (2005).

46. A. Elmaklizi, D. Reitzle, A. Brandes, and A. Kienle, "Penetration depth of focused beams in highly scattering media investigated with a numerical solution of Maxwell's equations in two dimensions," J Biomed Opt. 20, 065007 (2015).

47. J. Zheng, B. Yao, Y. Yang, M. Lei, P. Gao, R. Li, S. Yan, D. Dan, and T. Ye, "Investigation of Bessel beam propagation in scattering media with scalar diffraction method," CHIN OPT LETT. 11, 112601 (2013).

48. A. Aiello and J. P. Woerdman, "Theory of angular Goos-Hanchen shift near Brewster incidence," Physics, (2009).

49. W. C. Chew, Waves and Fields in Inhomogeneous media. (New York: IEEE Antennas and Propagation Society, Sponsor, 1995), p49. 